\title{
Clinicopathologic features of chronic nonspecific multiple ulcers of the small intestine
}

\author{
Motohiro Esaki $^{1} \cdot$ Junji Umeno $^{1} \cdot$ Takanari Kitazono $^{1} \cdot$ Takayuki Matsumoto $^{2}$
}

Received: 27 February 2015/Accepted: 6 March 2015/Published online: 19 March 2015

(C) Springer Japan 2015

\begin{abstract}
Chronic nonspecific multiple ulcers of the small intestine is a rare but distinct clinical condition, characterized by multiple small intestinal ulcers of nonspecific histology and chronic, persistent gastrointestinal bleeding without nonsteroidal anti-inflammatory drug use. However, because of the term "nonspecific" in its nomenclature, some gastroenterologists have misinterpreted the disease as the condition with small intestinal ulcers caused by undetermined etiologies without considering clinical features. Such misinterpretation has led to the heterogeneity of clinicopathologic features of the disease, as well as to ambiguity regarding a possible genetic contribution. It thus seems necessary to recognize the clinical entity of the disease precisely to avoid misinterpretation. In this review, we describe the clinicopathologic features, differential diagnosis, and the possibility of a genetic contribution to the disease.
\end{abstract}

Keywords Chronic nonspecific ulcer $\cdot$ Small intestine

\section{Introduction}

The clinical application of capsule endoscopy (CE) and balloon-assisted enteroscopy (BAE) has led to the possibility of encountering various small intestinal pathologies [1-3].

Motohiro Esaki

mesaki@intmed2.med.kyushu-u.ac.jp

1 Department of Medicine and Clinical Science, Graduate School of Medical Sciences, Kyushu University, Maidashi 3-1-1, Higashi-ku, Fukuoka 812-8582, Japan

2 Division of Gastroenterology, Department of Internal Medicine, School of Medicine, Iwate Medical University, Morioka, Japan
Among conditions manifesting small intestinal ulcers, Crohn's disease, intestinal tuberculosis, Behçet's disease, and nonsteroidal anti-inflammatory drugs (NSAIDs) enteropathy are conditions predisposing to chronic or recurrent small intestinal ulcers, while there still remain cases with unknown etiologies.

We have previously reported on an unusual form of enteropathy of unknown etiology, referred to as chronic nonspecific multiple ulcers of the small intestine (CNSU), which is characterized by chronic blood and protein loss through persistent small intestinal ulcers [4-6]. Although this rare but distinct clinicopathologic condition was initially reported in the Japanese literature by Okabe and Sakimura [7], the term "nonspecific" in its nomenclature which refers to the nonspecific histology of the ulcers has occasionally led to the misinterpretation of the disease as any condition with small intestinal ulcers of undetermined etiology. In Western countries, although the clinicopathologic features seem to be different from those of CNSU, Perlemuter et al. [8] have reported a similar enteropathy, termed "cryptogenic multifocal ulcerous stenosing enteritis (CMUSE)". In addition, a recent review considered CNSU to be identical to CMUSE [9]. Thus, precise recognition of the clinical entity of CNSU is mandatory to avoid misinterpretation. In this review, we describe the clinicopathologic features, differential diagnosis, and the possibility of a genetic contribution to the disease.

\section{Clinicopathologic features}

\section{Demographics, clinical symptoms, and laboratory data}

Table 1 summarizes the clinical features of 16 cases who were diagnosed as having CNSU. The disease predominantly 
Table 1 Summary of clinical characteristics of 16 patients with CNSU

\begin{tabular}{ll}
\hline 1. Gender & \\
Female:male & $13: 3$ \\
2. Age at onset ${ }^{\mathrm{a}}$ & $24.0[7-53]$ \\
3. Age at diagnosis ${ }^{\mathrm{a}}$ & $41.2[7-66]$ \\
4. Symptoms & \\
Anemia & $16(100 \%)$ \\
Abdominal pain & $6(38 \%)$ \\
Hypoproteinemia & $3(19 \%)$ \\
Edema & $1(6 \%)$ \\
5. Laboratory data & \\
Hemoglobin $(\mathrm{g} / \mathrm{dl})$ & \\
Serum protein $(\mathrm{g} / \mathrm{dl})$ & $9.1[4.8-11.2]$ \\
CRP (mg/dl) & $5.6[3.8-8.2]$ \\
6. Involved site & $0.4[0-1.6]$ \\
Stomach & \\
Duodenum & $5(31 \%)$ \\
Jejunum & $8(50 \%)$ \\
Ileum & $3(19 \%)$ \\
7. History of surgery & $16(100 \%)$ \\
Present & \\
Absent & $13(81 \%)$ \\
\hline
\end{tabular}

${ }^{a}$ Data are expressed as mean [range]

occurs in females, and the age at disease onset ranged from 7 to 53 years. Clinical symptoms of CNSU are mainly attributable to chronic and persistent blood loss from small intestinal ulcers. Patients usually manifest fatigue, edema, or abdominal pain, while they rarely complain of diarrhea, hematochezia, or fever. Although most patients have a history of long-term anemia, patients do not visit a gastroenterologist until long after disease onset because of their uncertain abdominal symptoms, resulting in a delay in the diagnosis of CNSU $[6,7,10]$.

Laboratory data also reflect persistent blood loss from the ulcers. Fecal occult blood tests are continuously positive and peripheral blood tests show hypochromatic and microcytic anemia. Most patients also manifest hypoproteinemia and hypoalbuminemia. However, acute inflammatory reactions, including C-reactive protein, $\alpha 1-$ and $\alpha 2-$ globulins, are usually within normal ranges or slightly increased [6, 7]. Leukocyte and platelet counts also are within normal ranges.

\section{Small intestinal findings}

The small intestinal ulcers in CNSU occur predominantly in the ileum, while the terminal ileum is usually intact [7, 10]. The ulcers are multiple (usually $>20$ ) and each lesion manifests a shallow and flat ulcer bed surrounded by a discrete margin.

The configuration of each ulcer is usually linear or a tall triangle, and the ulcer is aligned circularly or obliquely. The ulcers occasionally fuse, thus showing a geographic configuration. Such small intestinal lesions are radiographically depicted as multiple rigidity or eccentric deformities (Fig. 1a, b). Sharply demarcated barium flecks can also be depicted using compression study or doublecontrast barium study, although BAE is more suitable for evaluation of the shallow ulcers of CNSU (Fig. 2a, b). In
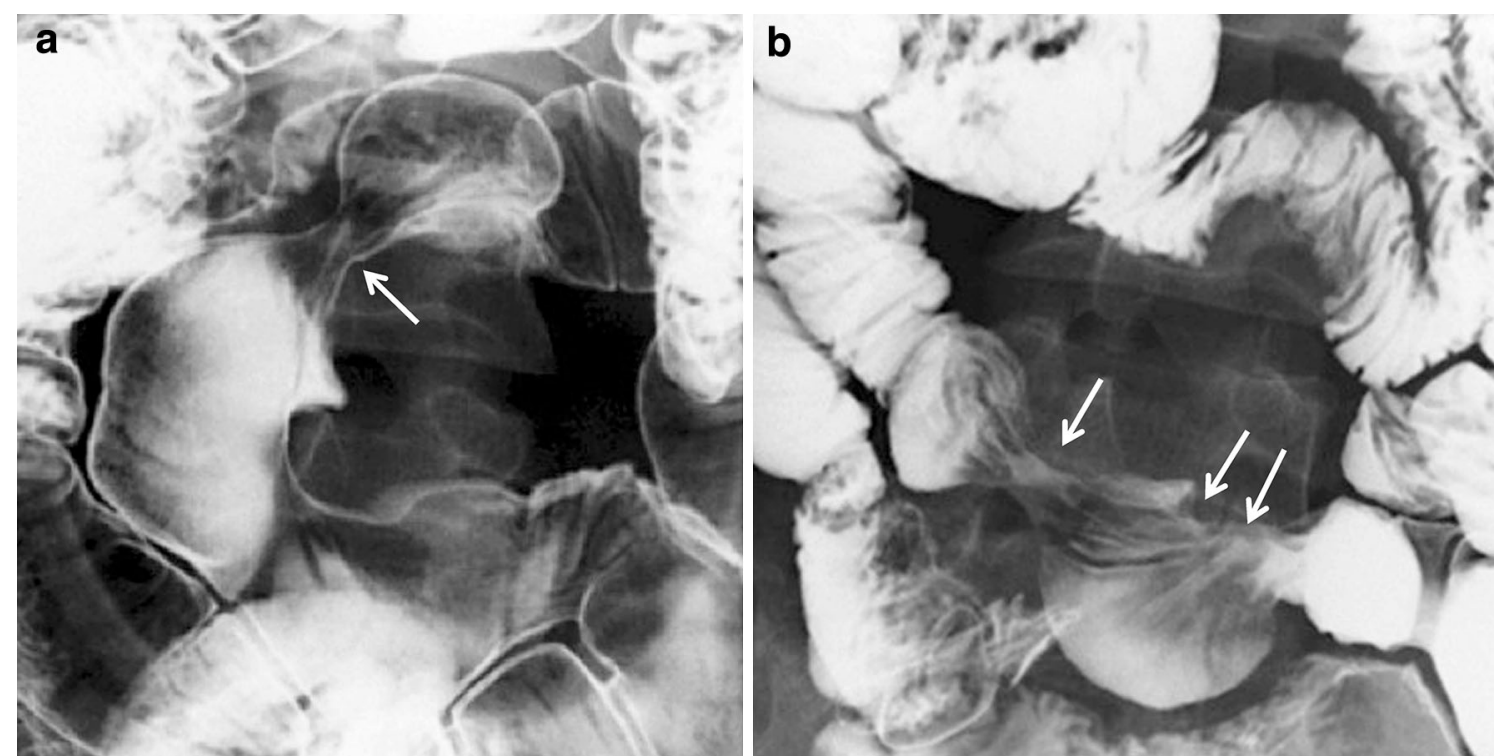

Fig. 1 Small intestinal radiographic findings. a Double contrast radiography depicts intestinal stricture (arrow) and eccentric deformities. b Sharply demarcated barium flecks (arrows) are depicted using compression study 

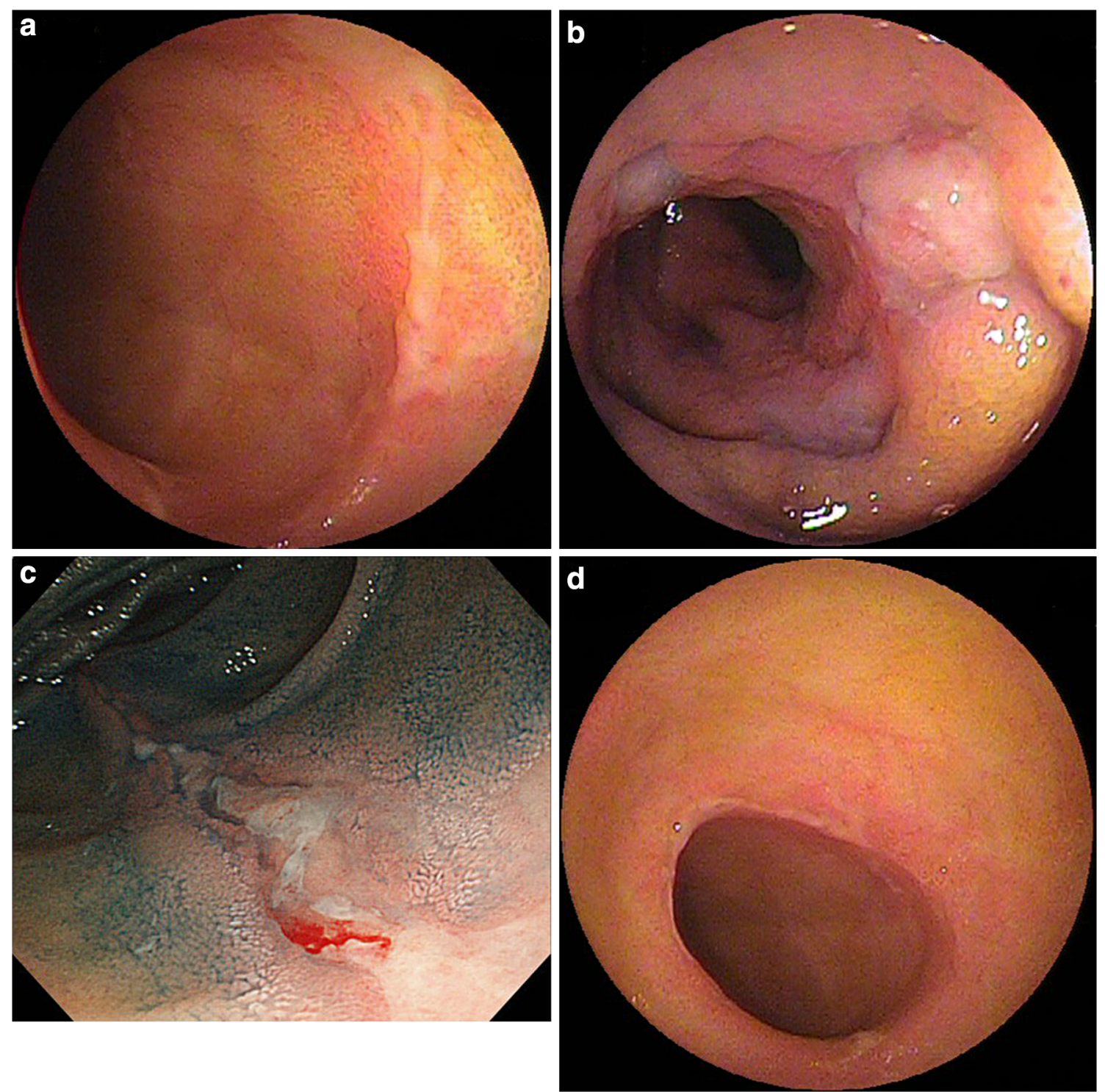

Fig. 2 Enteroscopic findings. Linear ulcer (a) and irregularly shaped shallow ulcers (b) are shown under retrograde BAE. c Contact bleeding from ulcer bed can be seen. d Intervening intestinal mucosa is apparently normal under enteroscopy

addition to the particular configuration and alignment of the ulcers, the ulcer bed is fragile and contact bleeding can occasionally be seen under enteroscopy (Fig. 2c). In contrast, the intervening mucosa is apparently normal without any diminutive lesions (Fig. 2d).

During the clinical course, the ulcers develop into intestinal strictures, mimicking the diaphragm-like strictures seen in NSAID enteropathy (Fig. 3a). However, because of the oblique nature of the pre-existing ulcers, the strictures are not always concentric but may show spiral patterns (Fig. 3b). CE should not be used due to the possible retention or impaction of the capsule. Small intestinal lesions of CNSU never progress to cobblestone appearance, fissure, or fistula formation or adhesion.
Although CNSU mainly involves the ileum, there have been cases of duodenal and colonic involvement $[11,12]$. Gastroduodenal involvement was found in 11 of our 16 cases; however, the clinical significance of these lesions needs to be clarified along with the status of Helicobacter pylori infection.

\section{Histologic findings}

The main histologic characteristics of CNSU are the depth and the healing process of small intestinal ulcers. The ulcer depth is restricted to the mucosa or the submucosa, and it never reaches the muscular layer $[6,7,10]$. The ulcer is clearly demarcated by surrounding villous mucosa, and 

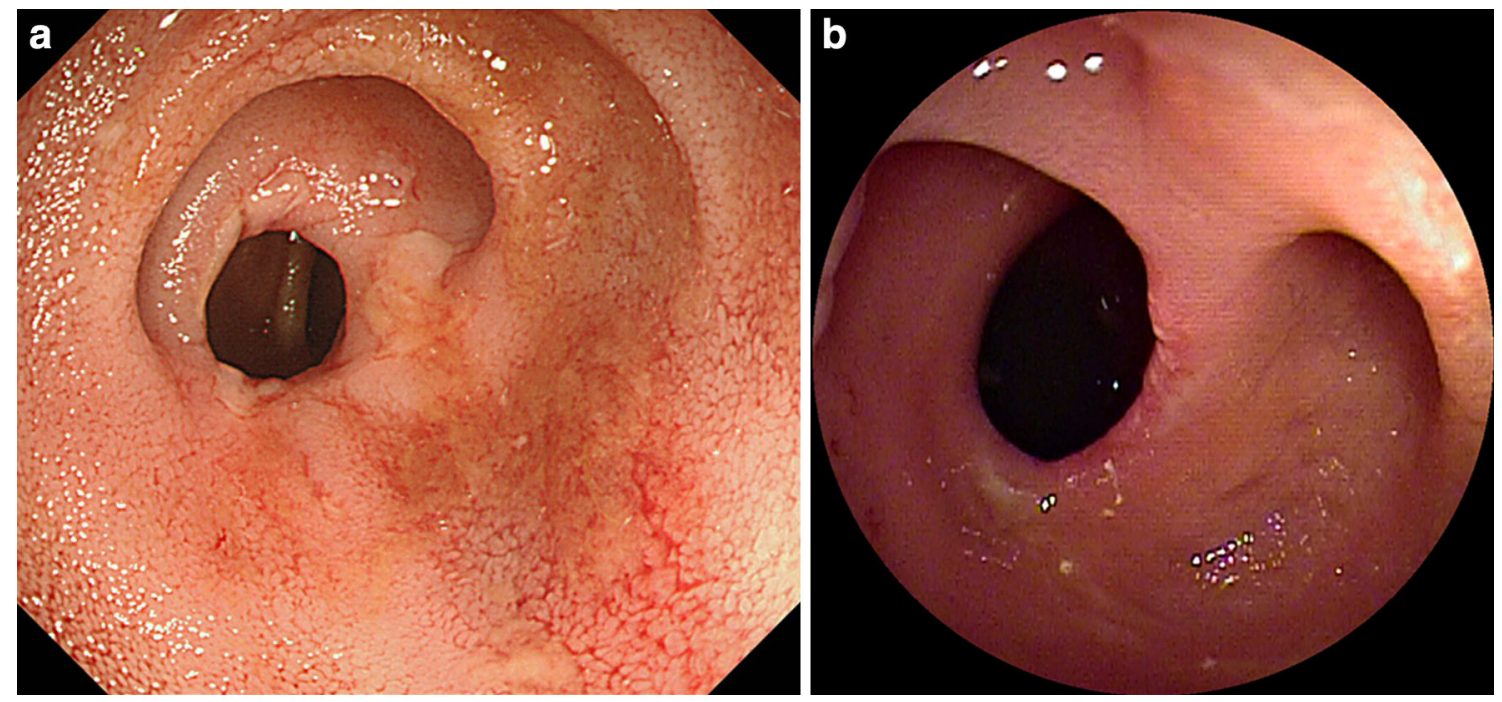

Fig. 3 Enteroscopic findings. a Concentric stenosis accompanied by shallow ulcers is found under intraoperative enteroscopy. b Spiral intestinal stricture is found under retrograde $\mathrm{BAE}$

only "nonspecific" chronic inflammatory cell infiltrates are found. In the healing process of the ulcer, submucosal fibrosis is restricted to the area of the mucosal defect with minimal epithelial repair and restitution, which has been referred to as the "ulcerative nonproliferative process" by Okabe and Sakimura [7, 10].

\section{Clinical course and therapies}

The clinical course of CNSU is characterized by the recurrence of small intestinal ulcers and stenosis even after surgery, because efficacious medical treatment strategy has not yet been established. Empirically, enteral nutrition coupled with iron supplementation can temporarily improve anemia and hypoproteinemia. Parenteral nutrition can also achieve mucosal healing. However, small intestinal ulcers and subsequent anemia and hypoproteinemia recur soon after the bowel rest is stopped. Previously, patients were obliged to undergo surgery when small intestinal stenoses occured, but balloon dilation under enteroscopy is now an alternative treatment for such complications. None of the medications used for inflammatory bowel diseases, including 5-aminosalicylic acid, prednisolone, and thiopurines, are effective for patients with CNSU [6, 7, 10, 11, 13]. In our experience, we have found no therapeutic effect of either thiopurines or anti tumor necrosis factor- $\alpha$ antibody. In addition, one patient received treatment with misoprostol which resulted in a failure of clinical improvement. Based on the above clinicopathologic features, a modified list of the diagnostic criteria of CNSU was proposed in 2004 (Table 2) [6, 14].

It has been recently revealed that certain extraintestinal manifestations can occur in CNSU (data submitted for
Table 2 Diagnostic criteria of CNSU

1. Persistent and occult blood loss from the GI tract except during bowel rest or postoperative period

2. Confirmation of characteristic small intestinal lesions by macroscopy, radiography, or enteroscopy

(a) Circular or oblique in alignment

(b) Sharply demarcated from surrounding normal mucosa

(c) Geographic or linear in shape

(d) Multiplicity in number with $<4$-cm distance from each other

(e) Ulcers not reaching proper muscular layer

(f) Scarred ulcers presumed to the healing stage of those characterized by (a)-(e $)^{\mathrm{a}}$ in cases treated by bowel rest

${ }^{a}$ Depicted as symmetric and eccentric rigidity under small-bowel radiography, and concentric or nonconcentric stricture under enteroscopy

publication); however, patients with CNSU are free from any complications, such as oral, skin, joint, genital, or perianal lesions, as are found in Crohn's disease and Behçet disease.

\section{Differential diagnosis}

Clinical conditions that can cause small intestinal ulcers with occasional strictures need be excluded.

Initially, Crohn's disease should be rigorously excluded, because both diseases share some common clinical characteristics: (1) susceptible during adolescence, (2) characterized by persistent anemia and hypoproteinemia, and (3) stricturing behavior of the small intestinal lesions $[15,16]$. In addition, there have been reports showing gastroduodenal and colonic involvement of CNSU [11, 12]. 
However, unlike Crohn's disease, the intestinal lesions of CNSU lack transmural inflammation, fissuring, fistula, and granuloma. Furthermore, the morphologic features of the small intestinal ulcers are completely different between the two diseases $[15,16]$. In conjunction with these histologic and morphologic differences, neither glucocorticoid nor thiopurines show any therapeutic effect in patients with CNSU $[6,7,10,11,13]$.

NSAIDs enteropathy is the second most important differential diagnosis of CNSU. This is because circumferential thin ulcers and concentric stenosis can occur in patients with long-term NSAID use [17, 18], and because the ileum is the predominantly involved site [19]. However, circumferential thin ulcers in NSAIDs enteropathy tend to occur on top of Kerckring folds and they are rarely aligned obliquely. Furthermore, the strictures can show spiral patterns because of the oblique nature of pre-existing ulcers in CNSU. Finally, NSAIDs enteropathy dramatically improves after the cessation of the causative drugs [4].

Intestinal tuberculosis is thought to be another differential diagnosis because mycobacterial species can cause chronic inflammation in the gastrointestinal tract developing multiple circumferential ulcers. However, the small intestinal ulcers in tuberculosis are characterized by an irregular margin and dense mucous exudates [7]. In addition, such ulcers are usually accompanied by multiple scarred ulcers and villous atrophy in the surrounding mucosa [7]. Finally, the ileocecal region is uniformly affected in patients with intestinal tuberculosis, and such patients will have a positive intradermal tuberculin test or interferon $\gamma$ releasing assay.

However, CMUSE, which has been mainly reported from European countries [8, 20-22], is another differential diagnosis. According to reports by Perlemuter et al. [8, 22], the clinicopathologic features of CMUSE are quite similar to those of CNSU. However, CMUSE has been suggested to be associated with vasculitis relating to heterozygous type I C2 deficiency [22]. In addition, CMUSE manifests extraintestinal manifestations (peripheral neuropathy, buccal aphthae, sicca syndrome, polyarthralgia, Raynaud's phenomenon, etc.), which are rarely seen in patients with CNSU [6, 7, 10, 11]. Finally, steroid therapy has a beneficial effect in CMUSE [8, 9]. Considering these differences in clinical condition, CMUSE should be assumed to be different from CNSU.

\section{Genetic contribution}

We have recently reviewed family histories of 13 patients with CNSU, and found 6 patients who were offspring of consanguineous marriage of $3^{\circ}$ or $5^{\circ}$. In addition, 3 of 13 patients had siblings showing enteropathy, and 2 of them were siblings of consanguineous marriage [23]. Based on the segregation in offspring from consanguinity, we speculated that CNSU is an autosomal recessive disorder [23]. In the present case series of CNSU, 8 of 16 patients were offspring of consanguinity marriages. In addition, 4 of the other 8 patients who denied consanguinity in their family pedigrees had siblings with CNSU. Such dense inheritance again confirms that CNSU is distinctive of hereditary disease.

Although CNSU is obviously different from NSAID enteropathy, both conditions share some similar morphologic features, where analogous etiology of the diseases can be postulated. In this context, Adler et al. [24] previously reported an unusual form of enteropathy with a life-long history of occult gastrointestinal blood loss causing iron deficiency anemia and relapsing abdominal pain. In middle-age, the patient manifested multiple, sharply demarcated ulcers and stenosis in the jejunum and the ileum, and histological examination of the resected specimens showed only nonspecific ulcers with minimal inflammatory infiltrates. In addition, the patient was confirmed to have inherited compound heterozygosity of the cytoplasmic phospholipase A2- $\alpha(c P L A 2 \alpha)$ gene, resulting in reduced eicosanoid biosynthesis in platelets and leukocytes. More recently, Brooke et al. [25] also demonstrated a homozygous deletion in PLA2G4A encoding cPLA2 $\alpha$ in patients with CMUSE by using genome-wide single nucleotide polymorphism homozygosity mapping combined with whole-exome sequencing. Since cPLA2 $\alpha$ catalyzes the release of arachidonic acid from membrane phospholipids, obviously impaired production of eicosanoids such as PGE2 and thromboxane A2 in CMUSE patients can cause multiple ulcers of the small intestine and platelet dysfunction $[24,25]$.

By using whole-exome sequencing, we have recently identified the gene responsible for the development of CNSU (data submitted for publication). Since the gene encodes a certain kind of protein that mediates intracellular prostaglandin levels in numerous tissues, aberrant function of the protein causes dysregulated prostaglandin levels in the intestinal mucosa, resulting in the development of multiple small bowel ulcers. Although the dysregulation of prostaglandins contributes to the pathogenesis of both CNSU and CMUSE, genetic analyses have revealed that the diseases are distinct clinical conditions.

\section{Conclusions}

In this review, we have described the clinicopathologic features, differential diagnosis, and the likelihood of a genetic contribution to CNSU. Our recent genetic analysis greatly contributes to the diagnosis of CNSU as well as 
differentiation from other clinical conditions showing multiple small bowel ulcers. However, since CNSU is a rare clinical condition, the accumulation of more patients is mandatory to further elucidate clinical, genetic, and pathophysiological characteristics of the disease. Eventually, effective medical therapy targeting the pathogenesis of the disease needs to be established in order to provide a better clinical outcome for patients with CNSU.

Acknowledgments This work was supported in part by Health and Labour Sciences Grants for research on intractable diseases from The Ministry of Health, Labour and Welfare of Japan.

\section{Disclosures}

Conflict of Interest: Motohiro Esaki, Junji Umeno, Takanari Kitazono and Takayuki Matsumoto declare that they have no conflict of interest.

Human/Animal Rights: All procedures followed were in accordance with the ethical standards of the responsible committee on human experimentation (institutional and national) and with the Helsinki Declaration of 1975, as revised in 2008(5).

Informed Consent: Informed consent was obtained from all patients for being included in the study.

\section{References}

1. Iddan G, Meron G, Glukhovsky A, Swain P. Wireless capsule endoscopy. Nature. 2000;405:417.

2. Yamamoto H, Sekine Y, Sato Y, et al. Total enteroscopy with a nonsurgical steerable double-balloon method. Gastrointest Endosc. 2001;53:216-20.

3. Yamamoto H, Kita H, Sunada K, et al. Clinical outcomes of double-balloon endoscopy for the diagnosis and treatment of small-intestinal diseases. Clin Gastroenterol Hepatol. 2004;2:1010-6.

4. Matsumoto T, Iida M, Matsui T, et al. Non-specific multiple ulcers of the small intestine unrelated to non-steroidal anti-inflammatory drugs. J Clin Pathol. 2004;57:1145-50.

5. Matsumoto T, Nakamura S, Esaki M, et al. Endoscopic features of chronic nonspecific multiple ulcers of the small intestine: comparison with nonsteroidal anti-inflammatory drug-induced enteropathy. Dig Dis Sci. 2006;51:1357-63.

6. Matsumoto $\mathrm{T}$, Iida $\mathrm{M}$, Matsui $\mathrm{T}$, et al. Chronic nonspecific multiple ulcers of the small intestine: a proposal of the entity from Japanese gastroenterologists to Western enteroscopists. Gastrointest Endosc. 2007;66:s99-107.

7. Okabe H, Sakimura MK. "Hi Tokui-sei Tahatsu-sei Shouchou Kaiyou-shou" (Nonspecific multiple ulcers of the small intestine). I to Chou (Stomach Intest). 1968:3:1539-49 (in Japanese).

8. Perlemuter G, Guillevin L, Legman P, et al. Cryptogenic multifocal ulcerous stenosing enteritis: an atypical type of vasculitis or a disease mimicking vasculitis. Gut. 2001;48:333-8.

9. Kohoutová D, Bártová J, Tachecí I, et al. Cryptogenic multifocal ulcerous stenosing enteritis: a review of the literature. Gastroenterol Res Pract. 2013;2013:918031.

10. Sakimura M. "Hi Tokui-sei Tahatsu-sei Shouchou Kaiyou-shou" no Rinshou-teki Kenkyu (Clinical study on "nonspecific multiple ulcers of the small intestine”). Fukuoka Igaku Zasshi. 1970;61:318-40 (in Japanese with English abstract).

11. Matsui T, Iida M, Kuwano Y, et al. "Hi Tokui-sei Tahatsu-sei Shouchou Kaiyou-shou" no Cho-ki Keika (Long-term follow-up study on non-specific multiple ulcers of the small intestine). I to Chou (Stomach Intest). 1989;24:1157-69 (in Japanese with English abstract).

12. Hoashi T, Matsui T, Takenaka K, et al. Ju-ni-shi-chou dai 2 bu ni Kaiyou-sei Byouhen wo Tomonatta "Hi Tokui-sei Tahatsu-sei Shouchou Kaiyou-shou" (Non-specific multiple ulcer of the small intestine accompanied by duodenal ulcer lesion: report of a case). I to Chou (Stomach Intest). 1991;26:1407-11 (in Japanese with English abstract).

13. Yao T, Fuchigami T, Sakimura M, et al. Chou no Kaiyou-sei Byouhen ni kansuru Atarashii Teian-Iwayuru "Hi Tokui-sei Tahatsu-sei Shouchou Kaiyou-shou" (A new proposal on ulcerative lesions of the small intestine-centering on so-called ulcerative, non-proliferative disease of the small intestine). I to Chou (Stomach Intest). 1972;7:1615-9 (in Japanese with English abstract).

14. Yao T, Iida M, Matsumoto T. Man-sei Shukketsu-sei Shouchou Kaiyou-Iwayuru "Hi Tokui-sei Tahatsu-sei Shouchou Kaiyoushou" (Chronic hemorrhagic ulcers of the small intestine or chronic nonspecific multiple ulcers of the small intestine). In: Yao T, Iida M, editors. Shouchou Shikkan no Rinshou (Diseases of the small intestine). Tokyo: Igaku-Shoin; 2004. p. 176-86 in Japanese.

15. Lennard-Jones JE. Classification of inflammatory bowel disease. Scand J Gastroenterol. 1989;24:s2-6.

16. Ueno F, Matsui T, Matsumoto $T$. On behalf of the guideline project group of the research group of intractable inflammatory bowel disease subsidized by the Ministry of Health, Labour and Welfare of Japan and the Gudelines Committee of the Japanese Society of Gastroenterology. Evidence-based clinical practice guidelines for Crohn's disease, integrated with formal consensus of experts in Japan. J Gastroenterol. 2013;48:31-72.

17. Matsuhashi N, Yamada A, Hiraishi M, et al. Multiple strictures of the small intestine after long-term nonsteroidal anti-inflammatory drug therapy. Am J Gastroenterol. 1992;87:1183-6.

18. Shumaker DA, Bladen K, Katon RM. NSAID-induced small bowel diaphragms and strictures diagnosed with intraoperative enteroscopy. Can J Gastroenterol. 2001;15:619-23.

19. Hayashi Y, Yamamoto H, Taguchi H. Nonsteroidal anti-inflammatory drug-induced small-bowel lesions identified by doubleballoon endoscopy: endoscopic features of the lesions and endoscopic treatments for diaphragm disease. J Gastroenterol. 2009;44(Suppl 19):57-63.

20. Shoesmith JH, Tate GT, Wright CJ. Multiple strictures of the jejunum. Gut. 1964;5:132-5.

21. Jeffries GH, Steinberg H, Sleisenger MH. Chronic ulcerative (nongranulomatous) jejunitis. Am J Med. 1968;44:47-59.

22. Perlemuter G, Chaussade S, Soubrane O, et al. Multifocal stenosing ulcerations of the small intestine revealing vasculitis associated with C2 deficiency. Gastroenterology. 1996;110:1628-32.

23. Matsumoto T, Kubokura N, Matsui T, et al. Chronic nonspecific multiple ulcer of the small intestine segregates in offspring from consanguinity. J Crohns Colitis. 2011;5:559-65.

24. Adler DH, Cogan JD, Phillips JA, et al. Inherited human cPLA (2alpha) deficiency is associated with impaired eicosanoid biosynthesis, small intestinal ulceration, and platelet dysfunction. J Clin Invest. 2008;118:2121-31.

25. Brooke MA, Longhurst HJ, Plagnol V, et al. Cryptogenic multifocal ulcerating stenosing enteritis associated with homozygous deletion mutations in cytosolic phospholipase A2- $\alpha$. Gut. 2014;63:96-104. 